\title{
Review
}

\section{Adhesion of microbes to the intestinal surface: lessons from the paradigm probiotic Lactobacillus rhamnosus GG}

\author{
Justus Reunanen ${ }^{1}$, Ingemar von Ossowski ${ }^{1}$, Willem M. de Vos ${ }^{1,2}$ \\ and Airi Palva ${ }^{1,3^{*}}$.
${ }^{I}$ Department of Veterinary Biosciences, University of Helsinki, P. O. Box 66, FIN-00014 Helsinki, Finland ${ }^{2}$ Laboratory of Microbiology, Wageningen University, $6703 \mathrm{HB}$ Wageningen, Netherlands
"These authors contributed equally
${ }^{3}$ Department of Veterinary Biosciences, Veterinary Microbiology and Epidemiology, P.O. Box 66, FIN-00014 University of Helsinki, Finland.

\begin{abstract}
The human gastrointestinal tract (GI-tract) is heavily colonized by a multitude of microbes, collectively called the microbiota. The microbiota provide us with metabolic capabilities not encoded in the human genome, e.g. the ability to utilize energy stored in dietary polysaccharides otherwise indigestible to us. Our microbiota also protects us against pathogens by competing for nutrients and binding sites within the GI-tract. Furthermore, given that cells of the GI-tract microbiota outnumber those of the host by ten-fold, this microbial ecosystem can be viewed as 'an organ in an organ'. The GI-tract microbiota has been a subject of intensive research during the last years, and its importance in health and disease is only starting to be realized because of recent breakthrough observations linking the human microbiota composition with major human diseases, such as diabetes and obesity. As the knowledge about the GI-tract microbiota composition and its impact on host health begins to accumulate, undoubtedly much of the research focus will shift from the systems level toward individual species and molecules. Consequently, there will be an increasing need for reductionist/functional proteomic approaches to identify and characterize the various 'molecular players' being utilized by different bacteria during their attachment to the intestinal epithelium of both healthy and diseased hosts. In this review we will highlight our recent findings about mucus adhesion mechanisms of the paradigm probiotic Lactobacillus rhamnosus GG.
\end{abstract}

Introduction

The intestinal epithelial cells (IECs) and the dendritic cells (DCs) are the cells that make most of the contact with microbes in the intestine. Much of the research on host-microbe interactions has been centered on the im-

\footnotetext{
* To whom correspondence should be addressed.

Phone : +358-9-191-57058

Fax : +358-9-191-57033

E-mail : airi.palva@helsinki.fi
}

munological aspects of these encounters, with the best understood examples being host pattern recognition receptors (PRRs) and microbe-associated molecular patterns (MAMPs). Despite the immunomodulatory effects caused by either commensals or pathogens, adhesion to host tissue is considered a crucial step in many host-microbe interactions, since the subsequent close association not only allows secreted factors contact with host tissue before they diffuse to the intestinal lumenand chyme, but also provides the prerequisite conditions for establishingstable colonization. In principle, bacteria can adhere 
either within the epithelia mucus layer or directly to IECs. In the small intestine, binding to IECs is often the case, where the mucus layer is discontinuous and many IECs are accessible to direct microbial contact, while in the colonthe mucus layer is continuous and so serves as the predominant site of adhesion. The mucus layer itself is a two-layered glycan-based coating of the epithelial cell lining of the inner intestinal walls, and is the largest surface area of the body available for contact with the external milieu ${ }^{28,35,43)}$. The highly hydrated mucus gel encompassing the outer layer of the intestinal mucosa consists of glycosylated membrane-bound and secreted mucins and serves as a protective barrier and filter ${ }^{21,2836}$. Consequently, the majority of intestine-dwelling bacteria have evolved surface-localized components with glycanrelated specificity to promote their adhesion to mucosal surfaces ${ }^{1}$. In addition to being merely a docking site for bacterial adhesion, the intestinal mucus also serves as a carbohydrate-rich nutrient source for 'professional mucus-eaters', such as Akkermansia muciniphila, which can survive using mucins as their sole source of carbon ${ }^{9}$.

To date, a variety of bacterial ligands that bind to host tissue have been identified. In lactobacilli, several different surface proteins have been shown to promote adhesion to mucus or epithelial cells ${ }^{15,19,22,31,38)}$, including S-layer proteins, sortase-dependent proteins, pili, and atypical surface proteins such as EFTu. In pathogens, a larger list of known examples exists ${ }^{8}$ 12, 42, 48. . However, while many of the described bacterial ligands that bind to host tissue are characterized from probiotic orpathogenic species, and even though the community structure of microorganisms encompassing the human intestinal microbiota is beginning to be resolved, there is still no detailed knowledge about the molecular mechanisms responsible for immunomodulation and host tissue adherence in human commensal bacteria. In this review, our focus will be on twotypes of surface adhesins common to many bacteria, namely pili and mucus-binding proteins, and herein, based on our recent findings, we will provide a detailed description about these adhesins in our favorite model microorganism, Lactobacillus rhamnosus GG.

Pili

Bacterial surface appendages that differ structurally from flagella were first described in the 1950s as a result of electron microscope imaging of the outer membrane of Gram-negative pathogens. These fibrils or rod-like structures were given two different names, because two research groups studied them simultaneously, one led by James Duguid, who designated the filaments as fimbriae (fringe) ${ }^{11}$, and the other directed by Charles Brinton, who called them pili (hair or fur) ${ }^{4,5)}$. Since then, these terms have been used interchangeably to describe non-flagellar appendages. In Gram-negative bacteria, where pili have been studied for many decades, the pilus backbone consists typically of homopolymers of noncovalently linked protein subunits ${ }^{39)}$. Interestingly, this type of pilus has also been found in few Gram-positive species, such as pathogens Corynebacterium diphtheria and Mycobacterium tuberculosis, and very recently in the human intestinal commensal Bifidobacterium breve, being encoded by the tight adherence $(t a d)$ locus and accordingly called the Tad pilus ${ }^{34}$. Contrastingly, the pili most commonly associated with Gram-positive bacteria are assembled as strings of pilin subunits, each of which are covalently coupled together by a recently discovered nonribosomal mechanism involving sortase-mediated catalysis $^{17}$. These sortase-dependent pili are structurally very slender, ranging only from 1 to $10 \mathrm{~nm}$ in diameter, and therefore difficult to detect and often overlooked in these organisms ${ }^{45}$. Thus far, sortase-dependent pili have been identified on the surface of several Gram-positive pathogens, including Corynebacterium diphtheriae ${ }^{13,44,46)}$, Actinomyces naeslundii ${ }^{30,52)}$, Bacillus cereus ${ }^{6)}$, Enterococcus faecalis $^{33}$, Enterococcus faecium ${ }^{16,17,18)}$, as well as the main invasive disease-causing streptococci in humans, Streptococcus agalactiae ${ }^{23)}$, Streptococcus pneumoniae ${ }^{2)}$, and Steptococcus pyogenes ${ }^{32}$.

Challenging the current doctrine that pili function primarily as pathogenicity factors, we recently identified the presence of pilus-like protrusions in Lactobacillus rhamnosus GG, a probiotic with well documented healthpromoting effects and a safe record of human consumption. Our observation of the piliated L. rhamnosus GG strain, which was confirmed by Western blotting and immunogold transmission electron microscopy (TEM), marked for the first time that multisubunit piliation (encoded by the spaCBA operon) could act as a novel mechanism by which probiotic LAB influence the intestinal health of the host ${ }^{22}$. Since then, gene clusters encoding for sortase-dependent pili have been found in few other non-pathogenic intestinal isolates, for example in Bifidobacterium longum subsp. longum ${ }^{20)}$. Interestingly, in comparison with the related L. rhamnosus LC705 strain, the SpaCBA pilus genes, encoding the SpaA major pilin and the $\mathrm{SpaB}$ and $\mathrm{SpaC}$ minor pilin subunits, are present in only L. rhamnosus $\mathrm{GG}^{222}$. Based on im- 
munogold single- and double-labeled TEM experiments using antiserum directed against each of the SpaCBA pilin subunits ${ }^{22.40}$, we have proposed a structural model for the macromolecular arrangement of the major and minor pilin constituents in the SpaCBA pilus ${ }^{40}$. In subsequent studies we revealed that purified recombinant forms of the $\mathrm{SpaB}$ and $\mathrm{SpaC}$ minor pilins both adhere to mucus from surgically resected human intestine. Additionally, evidence was also provided to suggest that the two minor pilin adhesins in SpaCBA pili (SpaB binding through electrostatic contacts and $\mathrm{SpaC}$ binding possibly in a lectin-type manner) are conceivably responsible for the adherence of $L$. rhamnosus GG cells to the intestinal mucosal surface ${ }^{22.49}$. Recognizing that there is no direct proof describing how probiotic lactobacilli occupy the GI tract, we surmised the presence of the SpaCBA pili with a mucus-binding capacity might explain the ability of L. rhamnosus GG to persist more stably in the intestine than other nonpiliated probiotic bacteria. For example, in comparison with $L$. rhamnosus GG, the genetically similar but non-SpaCBA piliated L. rhamnosus LC705 strain is significantly less adhesive to intestinal mucus ${ }^{47}$ and in a human intervention study is retained for a shorter length of time in the GI tract ${ }^{22}$. Additionally, recent work using knockout mutant derivatives of L. rhamnosus GG has suggested that the mucosal adhesion capacity of SpaCBA pili is a key component for promoting preferential adherence and biofilm formation in this probiotic strain $^{27}$.

\section{Mucus binding protein MBF}

Even though the SpaCBA pilus structures have so far been characterized as the predominant and preferential means for facilitating mucosal adhesion in $L$. rhamnosus GG, there are growing indications that other LPXTG-anchored cell wall proteins also have a functional role in the adherence of this probiotic strain to the host intestinal mucosa. For instance, a recent study ${ }^{37)}$ has reported that a L. rhamnosus GG knockout mutant for the LGG_01865 LPXTG-like protein (renamed as MabA for modulator of adhesion and biofilm) binds at least 2-fold less than the wild-type (WT) strain to intestinal Caco-2 epithelial cells, a cell line used commonly as an in vitro model of the human intestinal epithelium. However, in contrast to the overwhelming contribution of SpaCBA pili in mediating adhesion to mucus ${ }^{22,49}$, the MabA protein instead appears to merely modulate binding and likely has a secondary role in L. rhamnosus GG for ad- herence to intestinal cells ${ }^{37}$.

Recently, the three-dimensional structure of the type 2 MUB repeat of the $\sim 350-\mathrm{kDa}$ Mub (mucus binding) protein from Lactobacillus reuteri was determined ${ }^{29}$ and, besides a mucin binding property reported previously ${ }^{411}$, it was revealed that this domain repeat also has an affinity for several secretory immunoglobulins (SIg), including the IgA type. Given that SIgA antibodies are deposited regularly throughout the intestinal mucus layer and play a defensive role in preventing the establishment of invasive pathogens in host cells $^{7}$, it was suggested that Mub proteins with such a dual binding specificity would likely augment the overall intricacy of the adhesion mechanisms between some groups of intestinal bacteria, like lactobacilli, and the mucosal barrier ${ }^{29}$. However, in addition to mucus-specific SpaCBA piliation, we previously reported $^{22)}$ that the L. rhamnosus GG strain also contains one predicted surface protein that exhibits amino acid identity to a recognized mucus-binding domain ${ }^{3)}$. This putative mucus adhesin, which is genome-encoded by the LGG_02337 ORF and has been recently renamed the mucus-binding factor (MBF), is also encoded in the genome in L. rhamnosus LC705 (LC705_02328 ORF). Using recombinant-produced proteins, we showed that the adhesion capacity of MBF to human intestinal mucus is quite similar to that of the $\mathrm{SpaC}$ adhesin pilin when each was analyzed by the same microtiter plate-based assay method we had developed previously for assessing the mucus-binding properties of radiolabeled recombinant SpaCBA pilus proteins ${ }^{22,49,50)}$.

To achieve a better understanding of the role played by the surface-localized MBF in promoting adhesion between L. rhamnosus GG and the host intestinal mucosa, we examined the blocking effect of MBF-specific antibody on mucus binding. Accordingly, as a means to demonstrate MBF-mediated mucosal adhesion, we undertook antibody-mediated inhibition experiments in which cells of WT strains of $L$. rhamnosus GG (pretreated with anti-SpaC serum to eliminate the amount of mucus binding contributed by SpaCBA pili) and L. rhamnosus LC705 were treated with anti-MBF serum and assessed for adherence to intestinal mucus. Interestingly, when untreated LC705 cells or anti-SpaC antibody-treated WT $L$. rhamnosus GG cells were treated with anti-MBF serum, a statistically significant amount of mucus binding was abolished $\mathrm{d}^{50)}$. Clearly, the above results, together with our previous findings about pilus-mediated mucosal adhesion $^{22,50)}$, support the idea that the LPXTG-like MBF protein has a role, albeit less predominant, in the binding 
of the L. rhamnosus GG strain to the intestinal mucosa. Contrastingly, the MBF protein appears to be one of the main surface adhesins responsible for mediating the modest amount of adherence that exists between the $L$. rhamnosus LC705 strain and the mucus layer. By using L. rhamnosus GG MBF-specific antiserum in immunoblotting, we were able to reveal that MBF is available as a cell wall-associated surface protein in equal amounts in both L. rhamnosus GG and LC705 ${ }^{50)}$. However, despite this result, when each of these L. rhamnosus strains was examined with immuno-electron microscopy we could only detect $\mathrm{MBF}$ on the cell surface of LC705, but not GG. This seemingly evident discrepancy between the results obtained with two different methods using the same MBF-antiserum led us to consider, whether the the different make up of the exopolysaccharide (EPS) layers in these two L. rhamnosus strains ${ }^{22,25)}$ might be the reason for not observing MBF protein on L. rhamnosus GG cells. With that being said, when a L. rhamnosus GG knockout mutant (CMPG5351) lacking the long galactose-rich EPS layer was analyzed by immuno-electron microscopy for the presence of $\mathrm{MBF}$, we observedthat this derivative strain, like WT L. rhamnosus LC705, had also exhibited numerous $\mathrm{MBF}$ proteinson its cell surface ${ }^{50}$. We interpreted these results to suggest that the lengthy and more extended repeating units associated with the surrounding L. rhamnosus GG EPS layer might represent a physical obstruction that hinders the recognition of cell wall-bound MBF protein by the large-sized complexes of antibody molecules and protein A-gold particles.

\section{Conclusions}

Up to now, mechanistic insights about the exact features that distinguish the colonization ability amongst different intestinal inhabitants is still lacking and not fully understood ${ }^{51)}$, but for the most part are expected

\section{References}

1) Adlerberth, I., Cerquetti, M., Poilane, I., Wold, A., and Collington A.: Mechanisms of colonization and colonization resistance of the digestive tract. Microb. Ecol. Health Dis. Suppl. 2, 223-239 (2000).

2) Barocchi, M. A., Ries, J., Zogaj, X., Hemsley, C., Albiger, B., Kanth, A., Dahlberg, S., Fernebro, J., Moschioni, M., Masignani, V., Hultenby, K., Taddei, A. R., Beiter, K., Wartha, F., von Euler, A., Covacci, A., Holden, D. W., Normark, S., Rappuoli, R., and Henriques-Normark, B.: A to involve a variety of different interactions between cell surface adhesion molecules and the host intestinal mucosa. Lactobacillus rhamnosus GG, originally a human intestinal isolate that has over time become one of the most clinically investigated probiotic strains in commercial use today ${ }^{10)}$ and recently that has had its complete genome sequenced, serves as a paradigm probiotic for host-microbe interactions in the human intestinal context. Despite our prior work on the L. rhamnosus GG SpaCBA pilus and MBF, which includes mucus-binding ability and preliminary structural assessments, yet to be determined is a complete and detailed description of the regulation and concerted action of the different molecular adhesion mechanisms utilized by this bacterium within our intestine. As part of our prospective investigations on the functional and structural properties of L. rhamnosus GG SpaCBA-pili and MBF, we plan, by using recombinantproduced proteins, to identify and isolate their host cell receptors in the intestinal mucosa as well as undertake crystallization screenings for their 3D structure determination. Our ultimate goal is to achieve a structural glimpses of the adhesive interactions between the $L$. rhamnosus GG SpaC and MBF proteins and their mucosal target sites and, in so doing, provide a better understanding of the specific functional role played by these surface adhesins for probiotic colonization of the human intestine.

\section{Acknowledgments}

Financial support for the work described in this review was provided by research grants from the Academy of Finland. Docent Ilkka Palva is thanked for his scientific insights and comments during the course of this study. All authors are part of the Center of Excellence in Microbial Food Safety Research (MiFoSa) at the University of Helsinki.

pneumococcal pilus influences virulence and host inflammatory responses. Proc. Natl. Acad. Sci. U. S. A. 103, 2857-2862. (2006).

3) Bateman, A., Coin, L., Durbin, R., Finn, R. D., Hollich, V., Griffiths-Jones, S., Khana, A., Marshall, M., Moxon, S., Sonnhammer, E. L. L., Studholme, D. J., Yeats, C., and Eddy, S. R.: The Pfam protein families database. Nucleic Acids Res. 32, D138-D141. (2004).

4) Brinton, C. C. Jr.: The structure, function, synthesis and genetic control of bacterial pili and a molecular model for DNA 
and RNA transport in Gram-negative bacteria. Trans. NY Acad. Sci. 27, 1003-1054 (1965).

5) Brinton, C. C. Jr.: Non-flagellar appendages of bacteria. Nature 183, 782-786 (1959).

6) Budzik, J. M., Marraffini, L. A., and Schneewind, O.: Assembly of pili on the surface of Bacillus cereus vegetative cells. Mol. Microbiol. 66, 495-510 (2007).

7) Corthésy, B.: Role of secretory immunoglobulin A and secretory component in the protection of mucosal surfaces. Future Microbiol. 5, 817-829 (2010).

8) Costa, A. C., Figueiredo, C., and Touati, E.: Pathogenesis of Helicobacter pylori infection. Helicobacter 14(Suppl 1), 15-20 (2009).

9) Derrien, M., Vaughan, E. E., Plugge, C. M., and de Vos, W. M.: Akkermansia muciniphila gen. nov., sp. nov., a human intestinal mucin-degrading bacterium. Int. J. Syst. Evol. Microbiol. 54, 1469-1476 (2004).

10) Doron, S., Snydman, D. R., and Gorbach, S. L.: Lactobacillus GG: bacteriology and clinical applications. Gastroenterol. Clin. N. Am. 34, 483-498 (2005).

11) Duguid, J. P., Smith, I. W., Dempster, G., and Edmunds, P. N.: Non-flagellar filamentous appendages (fimbriae) and haemagglutinating activity in Bacterium coli. J. Pathol. Bacteriol. 70, 335-348 (1955).

12) Flores, J, and Okhuysen, P. C.: Enteroaggregative Escherichia coli infection. Curr. Opin. Gastroenterol. 25, 8-11 (2009).

13) Gaspar, A. H., and Ton-That, H.: Assembly of distinct pilus structures on the surface of Corynebacterium diphtheriae. $J$. Bacteriol. 188, 1526-1533. (2006).

14) Gorbach, S. L., and Goldin, B. R.: Lactobacillus strains and methods of selection. U.S. patent 4, 839, 281 (1998).

15) Granato, D, Bergonzelli, G. E., Pridmore, R. D., Marvin, L., Rouvet, M., and Corthésy-Theulaz, I. E.: Cell-surface associated elongation factor Tu mediates the attachment of Lactobacillus johnsonii NCC533 (La1) to human intestinal cells and mucins. Infect. Immun. 72, 2160-2109 (2004).

16) Hendrickx, A. P., Bonten, M. J., van Luit-Asbroek, M., Schapendonk, C. M., Kragten, A. H., and Willems, R. J.: Expression of two distinct types of pili by a hospital-acquired Enterococcus faecium isolate. Microbiology 154, 3212-3223 (2008).

17) Hendrickx, A. P., Budzik, J. M., Oh, S. Y., and Schneewind, O.: Architects at the bacterial surface - sortases and the assembly of pili with isopeptide bonds. Nat. Rev. Microbiol. 9, 166-76 (2011)

18) Hendrickx, A. P., Willems, R. J., Bonten, M. J., and van Schaik, W.: LPxTG surface proteins of enterococci. Trends Microbiol. 17, 423-430 (2009).

19) Hynönen, U., Westerlund-Wikström, B., Palva, A., and
Korhonen, T. K.: Identification by flagellum display of an epithelial cell- and fibronectin-binding function in the SlpA surface protein of Lactobacillus brevis. J. Bacteriol. 184, 33603367 (2002).

20) Iguchi, A., Umekawa, N., Maegawa, T., Tsuruta, H., Odamaki, T., Xiao, J. -Z., and Osawa, R.: Polymorphism and distribution of putative cell-surface adhesin-encoding ORFs among human fecal isolates of Bifidobacterium longum subsp. longum. Antonie van Leeuwenhoek 99, 457-471 (2010)

21) Johansson, M. E., Holmén-Larsson, J. M., and Hansson, G. C.: The two mucus layers of colon are organized by the MUC2 mucin, whereas the outer layer is a legislator of host-microbial interactions. Proc. Natl. Acad. Sci. U. S. A. 108 (Suppl 1), 4659-65 (2011).

22) Kankainen, M., Paulin, L., Tynkkynen, S., von Ossowski, I., Reunanen, J., Partanen, P., Satokari, R., Vesterlund, S., Hendrickx, A. P., Lebeer, S., De Keersmaecker, S. C., Vanderleyden, J., Hamalainen, T., Laukkanen, S., Salovuori, N., Ritari, J., Alatalo, E., Korpela, R., Mattila-Sandholm, T., Lassig, A., Hatakka, K., Kinnunen, K. T., Karjalainen, H., Saxelin, M., Laakso, K., Surakka, A., Palva, A., Salusjarvi, T., Auvinen, P., and de Vos, W. M.: Comparative genomic analysis of Lactobacillus rhamnosus GG reveals pili containing a human- mucus binding protein. Proc. Natl. Acad. Sci. U. S. A. 106, 17193-17198 (2009).

23) Lauer, P., Rinaudo, C. D., Soriani, M., Margarit, I., Maione, D., Rosini, R., Taddei, A. R., Mora, M., Rappuoli, R., Grandi, G., and Telford, J. L.: Genome analysis reveals pili in Group B Streptococcus. Science 309, 105 (2005).

24) Lebeer. S., Vanderlayden, J., and De Keersmaecker, S. C. J.: Host interactions of probiotic bacterial surface molecules: comparison with commensals and pathogens. Nat. Rev. Microbiol. 8, 171-184 (2010).

25) Lebeer, S., Verhoeven, T. L., Francius, G., Schoofs, G., Lambrichts, I., Dufrêne, Y., Vanderleyden, J., and De Keersmaecker, S. C.: Identification of a gene cluster for the biosynthesis of a long, galactose-rich exopolysaccharide in Lactobacillus rhamnosus GG and functional analysis of the priming glycosyltransferase. Appl. Environ. Microbiol. 75, 3554-3563 (2009).

26) Lebeer, S., Claes, I. J. J., Verhoeven, T. L. A., Vanderleyden, J., and De Keersmaecker, S. C. J.: Exopolysaccharides of Lactobacillus rhamnosus GG form a protective shield against innate immune factors in the intestine. Microb. Biotechnol. 4, 368-374 (2011).

27) Lebeer, S., Claes, I., Tytgat, H. L. P., Verhoeven. T. L. A., Marien, E., von Ossowski, I., Reunanen, J., Palva, A., de Vos W. M., De Keersmaecker, S. C. J., and Vanderleyden, J.: Functional analysis of Lactobacillus rhamnosus GG pili in re- 
lation to adhesion and immunomodulatory interactions with intestinal epithelial cells. Appl. Environ. Microbiol. 78, 185-193 (2012)

28) Liévin-Le Moal, V., and Servin, A. L.: The front line of enteric host defense against unwelcome intrusion of harmful microorganisms: mucins, antimicrobial peptides, and microbiota. Clin. Microbiol. Rev. 19, 315-337 (2006).

29) MacKenzie, D. A., Tailford, L. E., Hemmings, A. M., and Juge, N.: Crystal structure of a mucus-binding protein repeat reveals an unexpected functional immunoglobulin binding activity. J. Biol. Chem. 284, 32444-32453 (2009).

30) Mishra, A., A. Das, J. O. Cisar, and H. Ton-That. (2007). Sortase-catalyzed assembly of distinct heteromeric fimbriae in Actinomyces naeslundii. J. Bacteriol. 189, 3156-3165.

31) Miyoshi, Y., Okada, S., Uchimura, T., and Satoh, E.: A mucus adhesion promoting protein, MapA, mediates the adhesion of Lactobacillus reuteri to Caco-2 human intestinal epithelial cells. Biosci. Biotechnol. Biochem. 70, 1622-1628 (2006).

32) Mora, M., Bensi, G., Capo, S., Falugi, F., Zingaretti, C., Manetti, A. G., Maggi, T., Taddei, A. R., Grandi, G., and Telford J. L.: Group A Streptococcus produce pilus-like structures containing protective antigens and Lancefield $\mathrm{T}$ antigens. Proc. Natl. Acad. Sci. U. S. A. 102, 15641-15646 (2005)

33) Nallapareddy, S. R., Singh, K. V., Sillanpaa, J., Garsin, D. A., Hook, M., Erlandsen, S. L., and Murray, B. E.: Endocarditis and biofilm-associated pili of Enterococcus faecalis. J. Clin. Invest. 116, 2799-2807 (2006).

34) O'Connell Motherway, M., Zomera, A., Leahya, S., Reunanen, J., Bottacinia, F., Claesson, M. J., O’ Brien, F., Flynn, K., Casey, P. G., Moreno-Munoz, J., Kearney, B., Houston, A., O' Mahony, C., Higgins, D. G., Shanahan, F., Palva, A., de Vos, W. M., Fitzgerald, G. F., Ventura, M., O' Toole, P.W., and van Sinderen, D.: Functional genome analysis of Bifidobacterium breve UCC2003 reveals type IVb tight adherence (Tad) pili as an essential and conserved host-colonization factor. Proc. Natl. Acad. Sci. U. S. A. 108, 11217-11222 (2011).

35) Ouwehand, A. C., Salminen, S., Tölkkö, S., Roberts, P., Ovaska, J., and Salminen, E.: Resected human colonic tissue: new model for characterizing adhesion of lactic acid bacteria. Clin. Diagn. Lab. Immunol. 9, 184-186. (2002).

36) Patsos, G., and Corfield, A.: Management of the human mucosal defensive barrier: evidence for glycan legislation. Biol. Chem. 390, 581-590 (2009).

37) Perea Vélez, M., Petrova, M. I., Lebeer, S., Verhoeven, T. L. A., Claes, I., Lambrichts, I,. Tynkkynen, S., Vanderleyden, J., and De Keersmaecker, S. J. C.: Characterization of MabA, a modulator of Lactobacillus rhamnosus GG adhesion and biofilm formation. FEMS Immunol. Med. Microbiol. 59,
386-398 (2010).

38) Pretzer, G., Snel, J., Molenaar, D., Wiersma, A., Bron, P., Lambert, J., de Vos, W. M., van der Meer, R., Smits M. A., and Kleerebezem, M.: Biodiversity-based identification and functional characterization of the mannose-specific adhesion of Lactobacillus plantarum. J. Bacteriol. 187, 6128-6136 (2005).

39) Proft, T., and Baker, E. N.: Pili in Gram-negative and Grampositive bacteria - structure, assembly and their role in disease. Cell Mol. Life Sci. 66, 613-635 (2009).

40) Reunanen, J., von Ossowski, I., Hendrickx, A. P., Palva, A., and de Vos, W. M.: Characterization of the SpaCBA pilus fibers in probiotic Lactobacillus rhamnosus GG by immonogold electron microscopy. Appl. Environ. Microbiol (2012, in press).

41) Roos, S., and Jonsson, H.: A high-molecular-mass cell-surface protein from Lactobacillus reuteri 1063 adheres to mucus components. Microbiology 148, 433-442 (2002).

42) Sizemore, D. R., Roland, K. L., and Ryan, U. S.: Enterotoxigenic Escherichia coli virulence factors and vaccine approaches. Expert Rev. Vaccines 3, 585-595 (2004).

43) Sonnenburg, J. L., Angenent, L. T., and Gordon, J. I.: Getting a grip on things: how do communities of bacterial symbionts become established in our intestine? Nat. Immunol. 5, 569-573 (2004).

44) Swierczynski, A., and Ton-That, H.: Type III pilus of corynebacteria: Pilus length is determined by the level of its major pilin subunit. J. Bacteriol. 188, 6318-6325 (2006).

45) Telford, J. L., Barocchi, M. A., Margarit, I., Rappuoli, R., and Grandi, G.: Pili in gram-positive pathogens. Nat. Rev. Microbiol. 4, 509-519 (2006).

46) Ton-That, H., and Schneewind, O.: Assembly of pili on the surface of Corynebacterium diphtheriae. Mol. Microbiol. 50, 1429-1438 (2003).

47) Tuomola, E. M., Ouwehand, A. C., and Salminen, S. J.: Chemical, physical and enzymatic pre-treatments of probiotic lactobacilli alter their adhesion to human intestinal mucus glycoproteins. Int. J. Food Microbiol. 60, 75-81 (2000).

48) Valdez, Y., Ferreira, R. B., and Finlay, B. B.: Molecular mechanisms of Salmonella virulence and host resistance. Curr. Top. Microbiol. Immunol. 337, 93-127 (2009).

49) von Ossowski, I., Reunanen, J., Satokari, R., Vesterlund, S., Kankainen, M., Huhtinen, H., Tynkkynen, S., Salminen, S., de Vos, W. M., and Palva, A.: Mucosal adhesion properties of the probiotic Lactobacillus rhamnosus GG SpaCBA and SpaFED pilin subunits. Appl. Environ. Microbiol. 76, 20492057 (2010).

50) von Ossowski, I., Satokari, R., Reunanen, J., Lebeer, S., Keersmaecker, S. C. J., Vanderleyden, J., de Vos, W. M., and Palva, A.: Functional characterization of a mucus- 
specific LPXTG surface adhesin from probiotic Lactobacillus rhamnosus GG. Appl. Environ. Microbiol. 77, 4465-4472 (2011).

51) Walter, J.: Discovering the molecular foundations of Lactobacillus autochthony in the gastrointestinal tract. Jpn. J. Lactic Acid Bact. 19, 9-20 (2008).
52) Yeung, M. K., Donkersloot, J. A., Cisar, J. O., and Ragsdale, P. A.: Identification of a gene involved in assembly of Actinomyces naeslundii T14V type 2 fimbriae. Infect. Immun. 66, 1482-1491 (1998). 